\title{
La morale du but
}

Bruno Ackermann

\section{(2) OpenEdition}

Journals

Édition électronique

URL : https://journals.openedition.org/edl/1872

DOI : $10.4000 /$ edl. 1872

ISSN : 2296-5084

\section{Éditeur}

Université de Lausanne

\section{Édition imprimée}

Date de publication : 15 décembre 2019

Pagination : 123-146

ISBN : 978-2-940331-72-7

ISSN : 0014-2026

\section{Référence électronique}

Bruno Ackermann, «La morale du but », Études de lettres [En ligne], 311 | 2019, mis en ligne le 15 décembre 2021, consulté le 17 décembre 2021. URL : http://journals.openedition.org/edl/1872 ; DOI : https://doi.org/10.4000/edl.1872

Ce document a été généré automatiquement le 17 décembre 2021.

(c) Études de lettres 


\title{
La morale du but
}

\author{
Bruno Ackermann
}

1 La consultation des archives laisse apparaître la claire intention de Denis de Rougemont quant au livre qu'il voulait un jour achever. Il en a bâti la charpente, élaboré les arguments, anticipé les objections possibles. Il a mûri La morale du but pendant plus de trente ans. Ses abondantes notes en témoignent: une sorte de puzzle dont les pièces sont disséminées, parfois datées, et destinées à être intégrées dans différentes parties du manuscrit. Ce chantier d'écriture, qui a débuté en 1945, est séquencé par quelques périodes de travail et de relecture intense jusqu'en 1983, deux ans avant sa mort.

\section{Genèse d'une œuvre inachevée}

2 Ce projet naît à la fin de la guerre, dans « l'atonie générale ». Denis de Rougemont vit en exil aux États-Unis où il publie notamment La part du diable (1942), de nombreux articles de revue, rédige pendant quelques mois des bulletins de nouvelles à l'office of War Information. Depuis l'automne 1940, il voyage, déménage souvent, rien qui puisse assurer un travail d'écriture régulier. Amertume et découragement l'accablent :

Nous aurons peu pensé durant la guerre. [...] Nous aurons peu senti, peu réfléchi.

Nous attendions, dans la rumeur des commentaires et des regrets, et des vieilles

polémiques projetées sur un avenir très vague ${ }^{1}$,

note-t-il dans son Journal à la fin de l'année 1943. Pour autant, il s'agit pour l'écrivain de continuer son œuvre, d'écrire. La situation dramatique de l'Europe accapare son esprit. Les temps sont douloureux, les lendemains incertains :

La guerre "produit ", détruit, multiplie et divise (ce sont ses quatre opérations) mais ne crée rien. Sinon dans l'âme d'un peuple, par l'excès même de ses souffrances, un appel à des créations qui posent et garantissent pour les temps à venir les éléments d'un ordre humain. [...] Et qui donc parmi nous se soucie d'inventer ? ${ }^{2}$

4 Au printemps 1945 , les événements politiques s'accélèrent. La fin de la guerre est imminente. La conscience se réveille :

Flottant, isolé, menacé - c'est ainsi que je me sens et me décrirais ce soir. Pourtant, bon travail ces jours-ci, et confiance dans ce que je vais écrire (« Morale du But»). 
Un sens de l'ordre à instaurer et une vision du But, je ne les trouverai pas ailleurs :

il m'appartient de les... produire. Et peut-être pour moi... ${ }^{3}$

de bout en bout. Un premier dossier est constitué de feuillets manuscrits disparates, abondamment raturés, mais dûment numérotés et entre lesquels sont insérés des ajouts. Ce manuscrit se clôt par l'indication «The Porches, Lake George (N.Y.) 21 juillet-21 septembre $1945 »^{5}$. Dans ses archives, plusieurs dossiers, « Notes pour Morale du But $\|^{6}$, rassemblent des centaines de notes et feuillets, la plupart non datés, rédigés et amassés entre 1949 et 1983.

7 Un feuillet rédigé plus tardivement en guise de préface rappelle ses premières intentions :

$1^{\text {re }}$ version aux USA (Lake George) [pendant] la guerre [tout] de suite après [La] Part du Diable. Je me mouvais [dans] [une] soc[iété] [sans beaucoup] de traditions (comp[arée] à [1']Europe ou [l] Inde ou [la] Chine-Japon) où régnait en leur lieu le conformisme : ensemble de clichés ayant valeur de consignes : un h[omme] «vaut » tant (en \$), time is money, «dynamique », keep smiling, etc. - et de valeurs qui [sont des] clichés hérités de modes \pm récentes. D'où le sentiment de n'être pas situé [dans] un corps social, que j'éprouvais consciemment [plus] fort qu'eux, en tant qu'Europ[éen], [mais] qu'ils vivaient et subissaient plus intimement que moi. [...].

D'où [la] nécessité d'une théorie, d'une morale, capable de mieux assurer (ou regagner) le sens de l'identité (spécificité intime et globale) de l'individu, d'où la personne [?] par vocation « transcendante » par rapport à [la] Société .

Un autre feuillet, non daté, marque sa résolution d'écrire ce livre, non sans illusion, au regard du nombre de (vrais) lecteurs - ceux qui ont une « conscience réveillée » - que son essai pourrait intéresser :

Ce livre propose une morale radicalement contraire à ce que l'on croit, que «l'esprit du temps» appelle, mais dans cette mesure même elle correspond aux réalités de l'époque et répond à leurs vraies exigences. Celles-ci ne seront perçues que par très peu d'esprits. [...] Nous entrons dans l'époque des masses instruites, par là-même livrées à la publicité pour laquelle des auteurs de mon genre ne sont rien. Dans ce néant je trouve ma liberté, ma chance d'agir et d'être bien compris. Je propose une morale pour un seul contre les règles de la masse $[. . .]^{8}$.

La guerre, les camps, la bombe ont bouleversé la conscience humaine. De terribles convulsions politiques, économiques et sociales ont brisé toute foi en l'avenir. L'ère du doute paralyse l'esprit. Pourtant, sa vocation d'écrivain lui impose de participer à une réflexion en profondeur sur la place de l'homme dans la société :

Dans une société humaine de plus en plus unifiée, [...] j'écris pour résister à l'entropie, pour renforcer ceux qui voudraient croire à leur vocation individuelle et à leur aventure personnelle .

Le temps a manqué. Puis, les circonstances de la vie ont contrarié ses projets d'écriture, l'ont précipité vers d'autres engagements, plus urgents à ses yeux, notamment le combat pour une Europe fédéraliste. Une conférence aux Rencontres internationales de Genève en automne 1946 sur l'esprit européen marque un tournant dans son œuvre. Achever La morale du but n'eût pas été un problème en soi. L'amour et l'Occident a mûri 
durant toute son adolescence et sa jeunesse, puis a été rédigé en quatre mois. La morale du but a été "vécue » et "pensée " pendant de longues années. Difficile, pour nous lecteur de ce manuscrit inachevé, de mettre à sa place chacune des notes d'un livre à venir. Tout au plus peut-on ici éclairer les intentions de l'auteur, repérer les thèmes essentiels, les rattacher à l'œuvre dans son entier, et marquer ainsi la cohérence d'une pensée.

\section{Finalité, éthique, morale...}

Outre le manuscrit évoqué plus haut, La morale du but se présente sous la forme d'un tapuscrit de 109 pages, dupliqué en trois exemplaires, chacun corrigé différemment au gré de relectures espacées dans le temps. Il s'agit là du premier volume d'une œuvre qui devait compter deux parties, puis trois, puis quatre. Denis de Rougemont a souvent évoqué le projet d'un second volume intitulé Les règles $d u$ jeu ${ }^{10}$. Au titre initial - et le plus couramment repris dans ses notes -, d'autres titres possibles sont évoqués.

Ainsi Les finalités du but. Finalité et morale, si l'on se tient à la signification des termes, induisent des approches différentes. La finalité indique le fait d'avoir un but assigné, soit par une intention humaine ou personnelle, soit par une volonté supérieure, extérieure à soi. La morale infère des règles de conduite, des normes ou la recherche d'un idéal vers lequel tendre. La finalité est le but ultime d'un chemin, tandis que la morale est le cheminement, une longue marche existentielle où se dressent obstacles, difficultés et contingences humaines. La morale pose les repères concrets qui permettent d'atteindre un but, elle vise à éclairer l'homme dans la conduite de la vie. Trop souvent subsiste dans les croyances communes un grand malentendu : celui de confondre moyens et fins. Certaines visées humaines, immédiates et bien réelles, ne sont en réalité que des masques et des prétextes, non des fins en soi, lesquelles sont « invisibles à l'œil nu ».

Dans un entretien qu'il accorda peu de temps avant sa mort, Denis de Rougemont confiait alors que «toute action doit avoir pour fin l'homme, c'est à nous d'inventer l'avenir ", évoquant l'œuvre à laquelle il s'était attelé avec patience, voire avec opiniâtreté, La morale du but, justement, autrement nommé - troisième titre de l'œuvre projetée - La politique des finalités ${ }^{11}$. La politique est du ressort de l'action collective et sociale, de l'implication de l'homme dans la vie de la cité, davantage que de l'acte individuel. Une note témoigne de cet horizon plus large, cette dimension sociale et politique de la morale à laquelle Denis de Rougemont ne veut se soustraire :

Il me semble [...] que je n'ai parlé que d'une morale personnelle privée. Or la personne ne se réalise, n'existe que dans son actualisation communautaire (ne fût-ce que dans un livre écrit dans la langue d'une communauté). Il faut donc, nécessairement que la Morale du But comporte une morale du citoyen, une éthique politique, des aboutissements (prolongements) sociaux, civiques, politiques ${ }^{12}$.

Surgit ici une autre notion, celle de "l'éthique politique». Si l'éthique (science) et la morale (règles) ${ }^{13}$ sont étymologiquement synonymes et appartiennent au registre des mœurs, elles ne sont pas équivalentes, stricto sensu. La morale, du latin mores, s'inscrit, dès le début du XVIII ${ }^{e}$ siècle, dans une histoire culturelle de la modernité et des croyances religieuses et présuppose des convictions sur la distinction entre le bien et le mal, « des conceptions de l'acceptable ou du répréhensible dans les comportements humains $»^{14}$, d'où l'on déduit des règles de conduite, ou des normes, qui incitent à agir 
en fonction d'elles en tout temps et en tout lieu. L'éthique, du grec ethos, a une connotation plus réflexive, elle surgit d'un état d'intranquillité, elle induit un questionnement qui précède l'idée de morale avec une visée du bien dans un cadre politique et sociétal, rationnel et raisonnable.

Si Denis de Rougemont s'inscrit sans nul doute dans la lignée des grands moralistes du fait de son appartenance à la tradition protestante, affirmée et revendiquée, en disciple de Kierkegaard et de Karl Barth, il ne saurait cependant restreindre son champ d'analyse et d'action à cette seule dimension religieuse. À preuve, son premier essai, Politique de la personne (1934), le politique n'étant rien d'autre que l'affirmation de la personne dans le monde réel, la possibilité ou le choix d'agir dans le monde et de peser sur lui. Dans cet essai, l'éthique et la morale sont étroitement liées. Dans L'avenir est notre affaire (1977), l'éthique à coup sûr, dans un premier temps, l'emporte sur la morale par son questionnement sans concession sur l'avenir de la civilisation technicienne, par l'inquiétude qui se répand dans sa critique de la société contemporaine, puis rejoint la morale dès lors qu'elle suggère une politique afin de répondre à une décadence déjà largement amorcée : une morale définie comme un « ensemble de moyens d'adaptation d'une communauté à ses buts, une invention perpétuelle du chemin vers le But et l'improvisation vigilante qu'exige toute marche à l'étoile $»^{15}$. L'homme doit agir de manière responsable, non pour lui seul, mais pour le bien de tous, pour la planète. Dans ses livres consacrés à l'Europe et au fédéralisme, l'éthique prime sur la morale puisqu'il s'agit de bâtir des institutions politiques justes, qui permettent à l'homme de vivre en parfaite harmonie avec la cité, d'exercer une pleine responsabilité au sein de la communauté, d'imaginer des institutions qui sont à « hauteur d'homme $»^{16}$.

Ainsi, dans La morale du but, l'éthique et le politique ne sauraient être absents, ce sont les deux matrices inhérentes à son engagement intellectuel :

Il y a une politique dans ma morale du But.

La politique actuelle est dirigée (justifiée) par des principes absolutisés, des slogans pris pour axiomes, des proverbes, etc. Autant de «règles de conduites" individuelles appliquées à tort et à travers à des États, des nations, des classes, des groupes, des ensembles organisés, etc. [...].

Aucun de ces slogans absolutisés ne peut mener à la vraie fin de toute politique juste, qui est de permettre et d'harmoniser le libre développement des vocations personnelles, et dont la condition principale est le maintien de la paix. En revanche, la politique fédéraliste se déduit de cette fin et oblige à réviser tous ces slogans, dans la mesure où ils s'opposent à cette fin et ne peuvent servir que des fins nationalistes qui conduisent à la guerre et à l'oppression des vocations ${ }^{17}$.

17 Enfin, dans son essai magistral sur la conscience amoureuse en Occident, le mythe de la passion et la crise du mariage, le questionnement moral n'est pas absent. Denis de Rougemont y discernait deux morales, l'une héritée de l'orthodoxie religieuse, mais dénaturée et privée de foi vivante, autrement nommée la morale bourgeoise ; l'autre passionnelle et romanesque, inspirée par l'environnement culturel et littéraire. Il demeure que la morale, au sens rougemontien, est d'une part une continuelle adaptation des préceptes religieux à un contexte historique ou politique, structurée autant par des convictions personnelles que par une argumentation, et, d'autre part, dans la lignée de Karl Barth, que l'action de Dieu - la fin, le but ultime - prime sur l'action des hommes - le chemin pour l'atteindre, autrement dit les moyens. C'est le cœur même de sa philosophie personnaliste, de sa vision lucide du monde et de sa présence responsable. 
Quel qu'ait pu être le titre final retenu par Denis de Rougemont - La morale du but, Les finalités $d u$ but ou La politique des finalités, La fin de la morale ${ }^{18}$-, ce manuscrit, inlassablement remis sur le métier, procède d'une intention claire : donner sens, au fil du temps et de ses expériences, au regard d'un monde qui change et des profondes transformations de la condition des hommes dans ce siècle, donner corps à l'engagement de la personne au sein de la communauté humaine.

\section{Place de La morale du but dans l'économie de l'œuvre}

19 La morale du but n'est donc pas un livre né des seules circonstances évoquées plus haut: l'exil, la guerre, la bombe atomique, la crise de la conscience humaine. Les problèmes éthiques et politiques, la question des finalités ne sont ni des approches inédites ni marginales dans l'œuvre de Denis de Rougemont. Elles sont au cœur même de sa pensée, de sa volonté d'être au monde et de peser sur un monde en crise. Penser avec les mains (1936) procède déjà d'une analyse des transformations culturelles et politiques urgentes d'un monde où les idéologies totalitaires détruisaient et déshumanisaient l'Europe, brisaient l'aspiration des peuples à la liberté, anéantissaient leurs droits civiques et l'exercice d'une responsabilité personnelle.

20 Le chapitre II de la seconde partie de Penser avec les mains est une quête des « moyens d'action dont l'esprit de l'homme dispose " pour répondre à la crise, et contient déjà les "éléments d'une morale de la pensée ", préfigurant La morale du but. Il s'agit pour Rougemont de reconstruire une nouvelle "mesure » de l'humain au nom "d'une attitude finale ", c'est-à-dire d'un but ultime. La liberté de penser ne suffit plus, et même lorsqu'elle existe - quoique durant les années trente elle ait été collectivisée, embrigadée et manipulée -, elle doit être "libératrice », agissante, " pour que l'homme reste humain, ou le devienne ", au sens le plus absolu du terme. D'où l'injonction, la nécessité et la mise en œuvre d'une véritable révolution culturelle. L'agir doit suppléer la pensée, la pensée ne vaut rien sans l'acte concret qui transforme le monde et l'humain. La formule "penser avec les mains » est, à elle seule, ordonnatrice des "attitudes morales qui favorisent l'actualité de la pensée, qui en résultent, et qui en témoignent $»^{19}$.

21 Rougemont énumère ainsi les « vertus » ou les « valeurs » de cette morale audacieuse : réalisme, violence créatrice, autorité spirituelle, goût du risque, originalité, ascétisme de l'expression, imagination, style de vie, qui, toutes, n'ont d'autres sens que de faire retentir, au cœur de la réalité humaine, «l'appel des fins les plus lointaines », qui n'est autre que « l'acte à la fois immédiat et transcendant de la personne $»^{20}$. Autrement dit, sont posés ici quelques-uns des éléments annonciateurs de La morale du but. Dès les années trente, en pleine période de crise des valeurs et de la montée des périls en Europe, Denis de Rougemont cherchait déjà de nouvelles raisons d'espérer, "une direction toute nouvelle ", et aspirait à " mettre en œuvre au service d'un but nouveau une force intacte, endormie jusqu'ici $»^{21}$. L'après-guerre n'a fait que renforcer sa volonté de trouver un chemin original afin de dépasser les errements, les contradictions et les indécisions de l'homme moderne.

Ce manuscrit inachevé n'est-il dès lors que le prolongement de Penser avec les mains (1936), complément à Politique de la personne (1934), ouvrage dans lequel la question des " fins dernières ", « du destin de l'homme en face du destin du siècle » était déjà posée ? S'apparente-t-il à L'avenir est notre affaire (1977)? Dans cet essai sur la «crise de 
l'Avenir " en Occident, qui contient de nombreux éléments liés à sa philosophie personnaliste et communautaire, dans cette humanité confrontée à des réalités nouvelles - la dégradation des conditions environnementales et sociétales de la planète -, ressurgissent les mêmes interrogations fondamentales : « pour quoi ces efforts et vers quoi ? ", contraignant l'homme à choisir de manière libre et responsable son destin, le sien ou celui d'une civilisation qui pourrait mettre un terme à l'aventure humaine sur terre. Tous les ouvrages politico-philosophiques de Denis de Rougemont s'enracinent dans un "parti-pris ", avoué, revendiqué et manifesté clairement dès l'entame de ses livres. Écrivain engagé au sens le plus exigeant du terme, il met ses essais au service d'une fin dernière.

La morale du but pourrait être interprétée comme une relecture actualisée des changements culturels intervenus au sein de la communauté humaine. Cette œuvre en chantier revisite, de manière plus intime, les grandes interrogations sur le sens de la vie. "J'essayerai de dire ce que je crois ", écrit-il au détour d'une page, et non ce qu'il pense en tant qu'intellectuel. L'affirmation d'une foi est d'un autre ordre, elle ébranle l'être intérieur et force à l'examen de conscience : parole d'homme, engagement vis-àvis de soi.

\section{«Que faire de ma vie? »}

Son essai débute par une grande question métaphysique : «Que faire de ma vie?» Il porte sur le destin personnel d'un homme en quête de réponses en "un moment critique d'une ère de transition violente ", une après-guerre où tout est à (re)penser de fond en comble, et s'adresse à tout être humain soucieux de «mettre en question le sens et le but de [sa] vie dans ce monde ».

Cette "ère de transition violente" se manifeste, selon Denis de Rougemont, par différents symptômes: "l'invasion de l'arbitraire» dans la vie sociale, "la désorientation générale des individus", la perte de repères anciens qu'étaient les coutumes et les traditions, l'héritage familial ou le cadre culturel, «les voies d'une religion ou d'une foi efficace ", ou encore ces nouvelles formes de dictature que sont les modes, elles-mêmes fabriquées par des opinions publiques capricieuses, sans oublier les décrets administratifs et les ordonnances bureaucratiques qui réglementent la société, signes tangibles de la mainmise de l'État sur la conscience individuelle.

Tels sont les symptômes visibles du désordre, marqué par la dépersonnalisation de l'homme dépouillé dans ses choix, ou embarqué contre sa volonté par des contraintes extérieures. Les conséquences, par effet de convergence, sont doubles. D'une part ces réalités nouvelles ont tué «le sentiment de notre destination individuelle » et, d'autre part, ont nourri en chacun des hommes le goût de courir "son risque à sa guise ", sans lui permettre pour autant d'être sûr de sa vraie destinée ou porteur de certitudes nouvelles et personnelles. L'alternative est brutale, abrupte et implacable: «Trop de liberté ou point du tout; liberté vide ou liberté absente. Dans les deux cas, l'homme moderne est livré à l'angoisse de l'arbitraire ».

Nonobstant, Denis de Rougemont ne veut pas parler «contre l'époque », dont il subit fatalement les contingences, ni vanter un passé qui serait meilleur et vers lequel il faudrait se tourner. Il partage la condition de cette infinie diversité des hommes de son siècle. Sa lecture de l'histoire n'est pas réactionnaire. Point d'aspiration nostalgique à revenir aux traditions protectrices du passé et aux rassurants préceptes moraux, 
appelés eux aussi, pressent-il, à disparaitre. Même si les hommes du temps présent ne sont pas seuls à porter la responsabilité des échecs du passé et des dérives de la civilisation, il demeure acquis que le passé «a laissé se former ce présent détestable ». Dès lors, «il n'est jamais vain de voir clair et de comprendre », et «il n'est d'autre critique vraiment profonde d'un siècle que celle qui excite notre désir de l'aider à connaître son risque, à l'assumer, et à le dominer en création qui feront son honneur ».

Il s'ensuit une réflexion sur la croyance au progrès - cette idéologie héritée du XVIII ${ }^{e}$ siècle -, à laquelle d'aucuns attribueraient la cause des malheurs de l'époque. Encore convient-il de définir cette notion de manière exacte, à rebours de "l'optimisme des progressistes et $[\mathrm{du}]$ scepticisme des réactionnaires ", à l'opposé des régimes libéraux et des régimes totalitaires qui, tous deux, ont échoué dans l'édification d'une « humanité réelle ». Voici son hypothèse : « Le vrai progrès ne consisterait-il pas dans l'accroissement du risque humain?"

Tant la disparition et la destruction des coutumes, des règles et des structures héritées du passé que "l'angoisse de l'arbitraire ", qui dictent la démission de l'homme et ouvrent pourtant des chemins neufs, ces réalités engendrent un même péril, doublement inacceptable. L'alternative est simple : entre "l'incertitude insupportable " suscitée par un monde ouvert à la liberté avec sa part de risque, et «la contrainte insupportable » imposée par un monde totalitaire qui asservit l'homme, quelle issue inventer? «Une idée plus humaine de l'homme, une discipline personnelle ». Telle est $s a$ réponse. Cet axiome n'est pas inédit dans l'œuvre de Denis de Rougemont. Il s'agit de reconquérir et de réaffirmer un destin personnel face au « destin du siècle »" Mais il ne s'agit plus ici de l'affirmation de la personne, au sens général et philosophique du terme, mais davantage d'une volonté de forger une "morale personnelle», qui s'inscrive directement dans son expérience intime de la vie :

J'ai l'ambition, dans cet ouvrage, de formuler un principe de conduite, en pleine conscience de l'arbitraire où nous vivons, et tenter d'en surmonter le risque. [...] Mais je voudrais, à toutes fins utiles, verser au dossier de l'époque un exemple vécu de recherche et de solution personnelle ${ }^{23}$.

Non point formuler une ou des vérités générales pour les hommes de son temps, mais « m'expliquer - à moi-même d'abord ».

\section{La recherche du but}

31 Le récit minutieux de trois incidents militaires éclaire son propos. Curieuses mésaventures, en apparence seulement. Comme tout jeune citoyen suisse, Denis de Rougemont accomplit son service miliaire au cours duquel il apprend le maniement des armes, participe à une discipline collective qu'est la marche, vit enfin les affres de l'échec lors de l'une des marches. Ces trois expériences, apparemment anodines, lui ont permis de poser dans maints domaines la question de la «juste relation des moyens et des fins ». Quelles que soient les circonstances, les difficultés ou les contingences, seul l'appel du but détermine et oriente les ressorts (les moyens) de l'action, seule «la connaissance anticipée du but » insuffle à l'homme, souvent de manière insoupçonnée, la force et le courage, l'effort et l'énergie pour avancer. L'obéissance à certaines règles, préceptes légaux ou codes moraux, ne suffit pas à atteindre le but, et cette observance peut même s'avérer pernicieuse dans la mesure où elle détourne l'homme des "fins dernières ». Lorsque le but n'est pas atteint, alors l'échec s'interpose comme une 
«obscure sagesse " permettant à l'homme d'être ramené à son point de départ: la recherche du but et du sens des "visées humaines ».

Quels sont dès lors ces buts? Peut-on les expliciter, les classer, les hiérarchiser? L'infinie diversité humaine démultiplie les buts à foison, des plus triviaux et pratiques aux plus abstraits, subversifs ou imaginaires, spirituels ou indescriptibles. Ce qui importe pour Rougemont est d'abord de formuler les buts prochains de l'homme de son temps. Bien des buts, proches ou lointains - et les plus communs - ne sont, après analyse, que des moyens, "des masques et des prétextes» en vue de fins tout humaines: le gain d'argent, l'accumulation de richesse, l'ambition de s'instruire, la réussite professionnelle, les honneurs... Rougemont consacre cependant quelques feuillets à deux thèmes en particulier : le mariage et les idéaux politiques.

Du premier il retient que, s'il «n'est pas noué pour toute la vie, sans conditions ${ }^{24}$, les époux ne trouveront sans doute pas les forces ou la patience protectrice du couple « de ne point le briser ». Du but librement choisi et visé par chacun des conjoints dépendent les conduites humaines. Du second thème, à savoir les régimes politiques, il oppose la puissance des grandes idéologies totalitaires - le nazisme au but millénariste et le marxisme porté par «l'utopie toujours plus lointaine d'une société sans classe » - qui offraient certes en 1939 des buts lointains à la faiblesse des «démocraties opportunistes", lesquelles se contentaient d'une politique à "courte visée ». S'il entérine l'échec définitif du régime hitlérien, à savoir « cette impossible projection vers l'avenir d'un passé largement dépassé ", il accorde une rémission, toute provisoire, au régime communiste, porteur d'une utopie, d'une " contagion d'espoirs ». Mais dans un avenir proche, dit-il, ce régime prépare « la catastrophe majeure et la révolution la plus sanglante qu'aura jamais connue l'Histoire", pour la seule raison que le marxisme n'englobe pas le tout de l'homme, c'est-à-dire « une vision de ses fins et des options de base qui sont métaphysiques ». Or celles-ci ne peuvent être du ressort que des seules personnes, non de l'État. Et si les démocraties ont fini par l'emporter, c'est parce qu'elles ont fixé des buts plus grands que ces "utopies nationales et racistes », ou collectivistes. Dans les deux cas, le but lointain ou proche incitera les hommes politiques à agir différemment, à bâtir une "grande politique » qui «serait ordonnée au risque des personnes et de leur liberté».

La question demeure invariable : "Quel est le but de [la] vie? Quel est le sens même de la vie et de mon existence? » La plupart des hommes, observe Denis de Rougemont, "poursuivent des buts fous, j'entends: imaginaires ou impossibles, inavouables ou démesurés». Il existe autant de buts qu'il y a d'êtres humains, et rares sont ceux capables de répondre à cette simple question, par "peur d'être responsables", alors que d'autres "n'osent pas s'avouer qu'ils les recherchent ", fuient "devant les risques de la liberté, l'angoisse de se sentir une exception » ou se réfugient «dans la raison commune ». Les philosophies déterministes héritées des Lumières portent une lourde responsabilité, en Occident, « du vaste refoulement des valeurs spirituelles ».

L'un des buts prochains auquel Denis de Rougemont prête une attention particulière, parmi bien d'autres possibles, sont les "buts économiques", l'argent, une préoccupation qui touche de près ou de loin tout homme au cours de sa vie. Sur ce terrain apparemment solide, concret et quantifiable, les " lois économiques ", que vient démentir la psychologie humaine ou le surgissement d'une guerre, ces lois n'en gouvernent pas moins le monde et incitent l'immense majorité des hommes, par superstition ou par sottise, à les considérer comme des buts certains, et donc à ne pas 
remettre en question leur propre existence, en clair, à dénigrer « les buts spirituels au profit des buts mesurables ».

La science contemporaine ne demeure pas en reste dans ce débat; ses progrès sont incontestables et ont permis des avancées dans maints domaines, elle est utile, sérieuse, mais l'homme du XX ${ }^{e}$ siècle l'a érigée en méthode, dont les implications sont désastreuses :

Si nous prenons pour but de réduire le réel aux objets dont les «lois" [scientifiques ou économiques] paraissent nier l'esprit, notre sens spirituel s'atrophie et le monde se vide de significations ${ }^{25}$.

Sans doute la science n'est-elle encore qu'au seuil d'une évolution inimaginable et imprévisible. Une seule constante ressurgit avec force :

[...] la responsabilité de l'homme [...] Homme est ce qui, dans le cosmos, ne cesse et ne cessera jamais d'entendre des appels, [...] d'y répondre, ou de refuser d'y répondre ; ne cessera donc jamais de viser des buts au-delà de son état présent ${ }^{26}$.

Dès lors, la vraie valeur de l'homme ne s'apprécie que par ce que valent ses buts, se mesure à la grandeur des fins qu'il attache à sa vie personnelle ${ }^{27}$, voilà une première ébauche à la question première. Une autre interrogation demeure en suspens : où allons-nous? Tous les buts invisibles et lointains énoncés par Denis de Rougemont, qu'ils soient spéculatifs, mystiques, religieux, imaginaires ou politiques, nous ramènent toujours au même point, au but ultime : nous-même.

Un seul but peut être absolu : celui qui est au-delà de l'homme [...], au-delà de la fin des temps, dans l'Éternel présent et agissant ${ }^{28}$.

Tout ce qui témoigne de notre identité profonde et réelle dans ce monde: «le Transcendant » qui, Lui seul, détermine notre conduite, notre morale, le véritable point de départ de nos actes.

Le but détermine ainsi les moyens, les crée et les qualifie. L'adage, faussement attribué à Machiavel, pratiqué par les jésuites ${ }^{29}$ pour légitimer leurs crimes politiques, qui a attisé tous les fanatismes politiques $\mathrm{du} \mathrm{XX}^{\mathrm{e}}$ siècle, et contre lequel la croyance commune s'indigne, se heurte à trois erreurs aisément repérables : l'incompréhension, l'hypocrisie (la volonté de ne considérer que les «valeurs éternelles » pour justifier et légitimer la fin) et le dénigrement au nom de la loi divine, supérieure à toutes les autres. Ce qui justifie les moyens ne peut être que «la fin des fins". C'est de ce point qu'il faut concevoir et bâtir les conduites humaines, ordonner notre existence. Un autre adage courant, lui aussi contesté par la tradition et les vérités scientifiques, vient en soutien à cette quête : commencer par le commencement, autrement dit, se fonder sur «l'expérience humaine par excellence : la création». Denis de Rougemont en tire une conclusion en trois traits : la fin justifie les moyens s'ils concourent à une fin juste ; « une fin sans justice ne peut rien justifier »; enfin, « le jugement de bien ou de mal ne peut s'exercer sur les moyens qu'à partir des fins qui le dictent ». S'agissant de dissocier le bien du mal, les morales courantes, inculquées à l'individu par des traditions ancestrales, jugent parfois de manière hasardeuse ou trompeuse, soit au nom de certains principes immuables ou de valeurs éternelles, soit au nom de la «conscience morale » ou de la religion.

41 Pas plus l'Évangile n'a-t-il dicté des codes de conduite à ses fidèles, ni énuméré les méthodes et les vertus qui amèneraient le croyant à agir justement, ni prescrit la manière détaillée dont il devrait vivre ; il a dévoilé à l'homme un but ultime et invisible qui, seul, peut inspirer et conduire ses actions. Au risque de scandaliser les chrétiens, 
Rougemont affirme haut et fort que "l'Évangile n'est pas une morale, [qu'il] ne s'intéresse pas à la morale; qu'il n'en donne point ». Fi donc d'une morale chrétienne inspirée par la crainte de Dieu, réglementée par la tradition, l'éducation et les lois civiles ou les rites d'une communauté donnée. Sur ce point, Denis de Rougemont rejoint l'analyse de son maître en théologie, Karl Barth ${ }^{30}$.

Si la distinction du bien et du mal définie par rapport au seul but de l'homme, à savoir le "Transcendant ", a été tranchée, la question demeure irrésolue par rapport aux normes communautaires, où des réalités antinomiques, d'ordres divers, surgissent, et impliquent des choix, des attitudes permettant d'atteindre ce but. Ici la morale individuelle s'oppose à la morale collective. Toutes deux sont légitimes, valables, et l'une ne saurait être sacrifiée sans que l'autre n'en souffre gravement. La première est "luxe créateur", la seconde "nécessité conservatrice». D'où ce conflit permanent entre deux ordres de réalité inhérents à notre condition humaine :

Seul versus ensemble; anarchie versus coutumes; hic et nunc versus ubique et semper;

foi versus religion; inspiration versus rite; grâce versus loi; personne versus individu; violence [créatrice] versus sagesse, etc. ${ }^{31}$.

La morale sociale appartient à ce que Rougemont nomme les "règles du jeu », certes nécessaires au maintien d'une communauté humaine, à l'ordre politique, mais celles-ci comportent un danger : l'abandon de toute liberté et de toute cohérence personnelles. Prendre pour but de se conformer aux seules conventions sociales, à l'ordre établi au sein d'une communautés ${ }^{2}$ ou aux règles imposées par un groupe, c'est renoncer à soimême.

La réponse de Kant, dans ses Fondements de la métaphysique des mœurs - "agis dans chaque cas, comme si tout homme devait agir comme toi $»^{33}$ - ne saurait convenir pleinement à Denis de Rougemont. S'il adhère à la morale kantienne qui rend l'individu responsable envers l'humanité, «il a tort, dit-il, quant à la personne, qui est responsable devant Dieu et devant toute l'humanité de faire autre chose que tout le monde». La personne seule doit se rendre responsable de ses actes, elle seule peut découvrir son but, trouver son chemin et l'assumer réellement, "pour les autres et malgré eux ».

\section{Morale du but et vocation}

Dès lors, La morale du but ne saurait être qu'une morale personnelle, même si elle implique de manière inévitable des conséquences communautaires. Elle découle d'une vocation, thème récurrent dans tous les essais de Denis de Rougemont. La troisième partie du livre tente d'en explorer le sens profond, la manière dont tout homme la découvre ou l'invente, les effets et les conséquences qu'elle produit, les risques que cet appel provoque. L'intérêt de cette partie du livre est double, et formidablement inédite : d'une part, c'est la première fois dans le parcours intellectuel ou philosophique de Denis de Rougemont que le " phénomène » de la vocation, que ses maîtres à penser, Kierkegaard et Karl Barth, ont peu évoqué dans leurs écrits, ou du moins défini que dans la seule perspective chrétienne, est posé de manière aussi dense ${ }^{34}$; d'autre part, cette notion constitue la pierre angulaire de sa Weltanschauung, de la condition humaine dans le monde moderne. Par ailleurs, elle détermine sa quête intime et personnelle du but ultime, et donne sens à la question initiale : «Que faire de ma vie?» 
«Vocation $»^{35}$, du latin vocare, signifie appel. Et « tout appel signale un but, oriente vers lui, ou le désigne ». La manifestation de cette vocation peut prendre des formes différentes : vision, rêve, révélation, elle peut mûrir avec le temps, surgir au gré d'une expérience singulière ou, au contraire, être contrariée et même refoulée à jamais. La vocation n'est pas «la voix de la nature ", elle ne procède pas d'un choix rationnel et conscient. Rougemont cite quelques exemples d'hommes véritablement "appelés " (François d'Assise, T. E. Lawrence, Calvin) à devenir ce à quoi ils n'étaient pas destinés "par nature " et qui, soudain, se reconnaissent comme des êtres "sans précédent", littéralement transformés. Aux yeux d'autrui, la vocation est « incommunicable et donc invraisemblable ». L'homme qui a le courage de voir et d'accepter cet appel se distingue dès lors des autres, il devient unique, pleinement lui-même. La vocation n'oriente activement chaque être humain vers le but qu'à la condition qu'il réponde à cet appel par " un acte de dédicace ", autrement formulé une "déclaration du but » qui, seule, "fournit un critère de jugement pour tous les actes de celui qui l'énonce ». Or il est autant de buts qu'il existe d'hommes différents. Les actes qui en découlent « doivent être jugés d'abord en connaissance du but ", partant de soi et non au regard des morales courantes ou des coutumes en vigueur, car chaque homme « est seul à connaître son but ». Lui seul peut juger un acte bon ou mauvais en vue de la fin qui est la sienne.

Un autre aspect de la vocation est la relation que chaque homme entretient avec une communauté, de quelque grandeur ou nature qu'elle soit (le couple, le petit groupe, etc.). La multiplicité des buts humains peut engendrer l'anarchie, mais cet écueil s'évanouit dès lors que l'homme s'oriente vers le but dernier, contenu dans la formule décisive :

«Tu aimeras le Seigneur ton Dieu » - c'est le But, - « et ton prochain comme toimême » - c'est la Communauté, fondée sur la personne ${ }^{36}$.

La vocation relie des personnes libres et responsables, non des individus isolés et embrigadés dans les masses. Dans un monde où les doctrines politiques gouvernent la réalité collective et détournent de manière puissante l'homme de sa vocation première - être soi, totalement -, Rougemont rappelle que toute action sociale, lors même qu'elle peut s'avérer «morale » et fructueuse pour la société, ne peut être fondée que sur « la personne, son éducation et sa sauvegarde ».

La vocation indissolublement liée à la personne ${ }^{37}$, notion clé dans l'œuvre de Denis de Rougemont, est énoncée dès son premier essai. Les quelques feuillets de La morale du but consacrés à sa définition de la personne ${ }^{38}$ insistent toutefois sur l'idée que la vocation "ne peut être accueillie que par la personne même, qu'elle fait naître en l'appelant", autrement dit que la personne n'existe réellement que par l'appel impérieux et transcendant qu'elle reçoit, qu'elle n'existe qu'en acte, marquant ainsi son opposition aux philosophies rationalistes ou matérialistes.

Le chemin qui mène au but est dès lors "absolument vierge " puisque toute vocation est, par définition et par nature, unique, personnelle. D'où cette autre question: la vocation est-elle le fruit d'une découverte ou d'une invention? Philosophiquement, de nombreux auteurs, de Kant à John Rawls, ont marqué la différence entre ces deux notions, notamment dans le domaine de la science qui la distingue des autres activités humaines, la découverte étant la mise en lumière de quelque chose qui existe, l'invention, la création d'une chose ou d'une pensée qui n'existait pas auparavant. « La vocation étant l'appel du but ", l'homme ne peut y répondre que par une décision. La vocation ne peut être qu'une invention sans précédent, et induit, au cours de la marche 
vers le but, de nombreux risques : l'égarement - c'est-à-dire « l'erreur sur les voies et les moyens »-, l'épuisement - ou le doute, une «forme de fatigue spirituelle »-, la paresse d'esprit et la lâcheté, qui sont autant de faiblesses inhérentes à notre humaine condition, et ces obstacles s'avèrent d'autant plus réels et même nécessaires que le but est grand et lointain. Impossible, par avance, de vérifier si le chemin choisi mène au but. Seule la foi, définie comme « substance des choses espérées » par saint Paul (Épitre aux Hébreux, 11.1), permet à l'homme, dans son infinie solitude, de surmonter toutes les épreuves qui surgissent sur son chemin. Denis de Rougemont se réfère explicitement aux épittres de saint Paul qui distinguait les trois stades de l'existence humaine, ainsi résumée :

L'homme qui vivait sans loi, dans l'arbitraire ; l'homme qui vit sous la Loi, dans la contrainte ; l'homme qui vivra par la grâce et la foi, dans la libertée ${ }^{39}$.

51 Le premier stade évoqué dans les textes pauliniens évoque un monde régi par la tyrannie; le deuxième stade, un monde régenté par les morales ambiantes, les règles coutumières, les modes ou les «élans passionnels déclenchés par l'État »; le troisième, enfin, ce monde neuf et vivant d'une vocation reçue et obéie.

Sans tomber dans un idéalisme que d'aucuns lui reprocheraient à coup sûr, Denis de Rougemont croit "au pouvoir de l'imagination ", aux facultés créatrices de l'homme qui, avec la foi, sont porteuses d'une vision lointaine. Pour rejoindre un but, il importe que la vocation confère à l'homme une manière d'être, c'est-dire un "style de vie $»^{40}$, qui est «l'une des marques distinctes de la personne en exercice». Le "style de vie » est une "pesée constante» de cette lutte que la personne doit mener contre les coutumes et les lois existantes. La personne, du fait de sa vocation qui la distingue et l'isole de tous, est inexorablement confrontée à un fatras d'obstacles de tous ordres, matériels, institutionnels, sociaux et politiques, voire psychologiques. Ainsi par exemple celui de l'égalitarisme, une «obsession» commune aux régimes démocratiques et aux systèmes totalitaires, en tant que cette hantise est un barrage à l'affirmation des vocations, une forme d'intolérance à l'égard de la différence :

Égaliser, centraliser, [unifier], telles sont [les] devises [du $\mathrm{XX}^{\mathrm{e}}$ siècle] : or ce sont celles de la paresse intellectuelle, de la débilité de l'imagination, et du mépris de la personne $e^{41}$.

53 À ces «[adversaires] de l'humanité ", Denis de Rougemont répond: "liberté et fédération ». Enfin, dernier danger qui guette l'époque, le "refoulement systématique des vocations ", à moins que l'homme ne libère les énergies suscitées par le but qu'il a vu ou imaginé, et ne se mette en marche qu'avec «le sentiment d'une liberté enfin réelle et orientée ".

La libertée ${ }^{42}$ devient dès lors le maître-mot pour franchir les obstacles. Tout homme détient cette faculté de choisir librement ses fins, de les accepter, de les réaliser et d'y conformer ses moyens. Mais soudain terrorisé par l'idée d'exercer pleinement cette liberté, c'est-à-dire de manière responsable, l'individu invente des «lois » et des coutumes qui, littéralement, l'empêchent de choisir librement son destin, préférant le confort d'une existence mutilée et routinière, imposée par la bien-pensance. «Il n'y a de loi que là où l'homme renonce à se manifester selon sa vocation personnelle", écrivait-il ailleurs ${ }^{43}$. Or, l'appel du but précipite l'homme « dans le vif d'un drame bien noué », et l'oblige, contre son gré, ses goûts ou ses inclinations naturelles, à choisir une liberté nouvelle qui seule permet l'exercice de sa vocation. De nombreuses libertés se heurtent au monde réel; ainsi de la « liberté civique », qui «n'est pas de celles que l'État puisse 
jamais proposer », ou de la « liberté sociale " (l'ensemble des libertés individuelles). Si ces libertés ne proviennent pas de la personne même, l'État les dictera à l'ensemble du corps social. Cette mécanique est connue, elle engendre l'État totalitaire que Denis de Rougemont dénonce sans fatigue depuis longtemps, reprenant ici une formule énoncée ailleurs : «Là où l'homme veut être total, l'État ne sera jamais totalitaire " ${ }^{44}$.

L'éducation est l'une des solutions possibles, souhaitables, pour remédier aux entraves qui pèsent sur l'élan individuel vers l'exercice de la vocation. Loin d'être limitée à la seule activité de transmission et d'inculcation des connaissances et des « règles du jeu », une tâche qui demeure malgré tout indispensable au maintien d'un contrat social minimal, l'éducation doit dans le même temps inspirer un élan original : «le courage d'aller au-delà ", de rompre avec certains codes moraux, préjugés, modes de penser, "vérités reçues", etc. Telle est la liberté de l'homme, de conduire sa vie selon un rythme personnel, un "style de vie » qui suppose une «maitrise de soi en souplesse » en vue d'un but "ambitionné ». Là encore, l'exercice de la liberté personnelle se heurte aux jugements moraux d'autrui, ceux-là mêmes dictés par la morale chrétienne, la morale bourgeoise ou «la morale du temps». Denis de Rougemont reprend à son compte, comme souvent, des versets bibliques pour résoudre cette épineuse question : « Ne jugez point ", tel est le commandement. Or, il est dans la nature humaine de juger de tout, en dépit de tout. Les répliques de Denis de Rougemont sont alors cinglantes : tout jugement moral sur le prochain est "par essence non-chrétien », car ne mérite l'adjectif chrétien que ce qui " relève de la foi seule, toujours liée au doute »; tout jugement moral relève "d'une vision faussée", car nul ne peut connaître le but d'autrui, discrédite le prochain, excite la jalousie ; enfin, tout jugement relève de l'anxiété, fruit d'une mauvaise conscience qui se manifeste, entre autres caractères ou attitudes, par la méchanceté, l'aigreur ou la sottise, l'ignorance ou le fanatisme. Il en est de même pour toute autre forme de jugement, sur le plan théologique, politique ou intellectuel. La seule question qui vaille, pour Rougemont, est le but visé. Au reproche de relativisme que d'aucuns lui adresseraient, il répond sèchement: "Essayez de vous juger vousmême au nom du but que vous servez ».

Le dernier petit chapitre qui clôt La morale du but, « Pour un seul », appelle à une morale personnelle afin de répondre en conscience aux tourments de notre époque, un défi auquel l'homme n'a jusqu'ici pas été confronté au cours de l'histoire. Tous les problèmes de notre temps n'auront jamais été aussi complexes, et toutes les réponses n'auront jamais été aussi vaines, sinon dérisoires au regard des dangers mortels qu'encourt l'humanité. Or, dit-il, « entre le drame de la personne et le destin mécanique du siècle, si étroitement liés, si étrangement complices, je n'ai plus trouvé place pour les morales anciennes, ni pour une morale renouvelée qui s'organiserait en système de valeurs, de vertus, de jugements sur les moyens en soi ». Loin de vouloir imposer une morale à autrui, il appartient à l'homme, en lui-même, de chercher et de trouver une orientation personnelle à sa vie, que résume le terme de vocation.

\section{Conclusion}

57 Aussi inachevé qu'il soit, cet essai n'en révèle pas moins les concepts clés de la pensée de Denis de Rougemont, des liens étroits avec d'autres œuvres publiées de son vivant. Mais surtout une pensée toujours en mouvement, jusqu'au dernier instant, forgée par une conviction inébranlable dans sa mission d'écrivain et de penseur, soucieux 
d'interroger et d'instruire - au sens étymologique de ce terme, soit « former l'esprit » les hommes de son temps. Une "morale de la vocation", non point une morale générale du genre humain, mais un chemin sans précédent qui conduise chacun des hommes, de manière libre et responsable, à la source d'un but librement choisi et accepté. La morale du but répond-elle à sa question : «Que faire de ma vie? » Sa vocation d'écrivain, de son propre aveu, a consisté davantage à « poser des questions qu'à tenter d'imposer des réponses : à poser avant tout, en temps et hors du temps, la Question, celle du Sens, celle du But $»^{45}$.

\section{BIBLIOGRAPHIE}

BARTH, Karl, Parole de Dieu et parole humaine, Paris, Je Sers, 1933.

—, « La vocation de l'homme », Dogmatique, vol. IV, t. III/II, Genève, Labor et Fides, 1973.

BONHOEFFER, Dietrich, Éthique, Genève, Labor et Fides, 1949.

KANT, Emmanuel, Fondements de la métaphysique des mœurs, trad. Victor Delbos, Paris, Librairie Delagrave, 1974

MASSÉ, Raymond, Anthropologie de la morale et de l'éthique, Laval, Presses de l'Université Laval, 2015. RICOEUR, Paul, Soi-même comme un autre, Paris, Éditions du Seuil, 1990.

ROUGEMONT, Denis de, « Définition de la personne », Esprit, (1 ${ }^{\mathrm{er}}$ décembre, 1934), p. 368-382.

—, « Destin du siècle ou destin de l'homme », in L'ordre nouveau, 11 (15 mai 1934), p. 3-7.

—, « Grammaire de la personne », Hic et Nunc (janvier 1934), p. 18-23.

—, Politique de la personne, Paris, Je Sers, 1934.

-, Penser avec les mains, Paris, Albin Michel, 1936.

—, « La vraie défense contre l'esprit totalitaire », Les Cahiers protestants, 7 (1938), p. 411-425.

—, L'amour et l'Occident, Paris, Plon, 1939.

—, Mission ou démission de la Suisse, Neuchâtel, La Baconnière, 1940.

-, « Pour une morale de la vocation », in Le problème éthique à l'heure actuelle, Neuchâtel, Société pastorale suisse, 1966 , p. 17-33.

-, Journal d'une époque, Paris, Gallimard, 1968.

-, L'avenir est notre affaire, Paris, Stock, 1977.

—, « Denis de Rougemont tel qu'en lui-même » [entretien], Cadmos, 33 (printemps 1986), p. 7-23.

SAINT-OUEN, François (éd.), L'Europe de Denis de Rougemont, Publications de l'Institut européen de l’Université de Genève, 2014. 


\section{NOTES}

1. D. de Rougemont, Journal d'une époque, p. 548.

2. Ibid., p. 548.

3. Ibid., p. 553 sq.

4. Les deux premières lettres sont datées des 8 et 12 août, Lake George.

5. Fonds Denis de Rougemont, Bibliothèque publique et universitaire de Neuchâtel [désormais Fonds DdR, BPUN], « L'arbitraire, ou le risque de ce temps », ms., 54.2.2. (107 ff.).

6. Fonds DdR, BPUN, «La morale du but », 54.1.1. et suivants, 54.2.1. et 54.2.3. Le projet est apparemment plus ancien. Un feuillet intitulé « Morale du but », dans le dossier 54.2.3 indique la date du 21 juin 1944, en cinq chapitres.

7. «Pour une préface », 20 février 1981, ms. Fonds DdR, BPUN, 54.1.4.

8. Préface, ms. s.d. Fonds DdR, BPUN, 54.1.1.

9. Note ms. s.d., intitulée « Point de départ », Fonds DdR, BPUN, 54.1.1.

10. Projet esquissé au printemps 1942. Sur un plan de travail en vue d'un fellowship financé par la Bollingen Foundation, cf. Journal d'une époque, p. 507-511.

11. «Denis de Rougemont tel qu'en lui-même », p. 20 sq. L'entretien date de fin septembre 1985.

12. Feuillet intitulé « Adjonction importante 28 décembre $1977 »$ (Fonds DdR, BPUN, 54.1.4). C'est Rougemont qui souligne.

13. Sur cette distinction, cf. les pages éclairantes de Paul Ricœur qui réserve le terme d'éthique "pour la visée d'une vie accomplie ", et le terme de morale "pour l'articulation de cette visée dans des normes " (Soi-même comme un autre, p. 200).

14. R. Massé, Anthropologie de la morale et de l'éthique, p. 21 ss.

15. L'avenir est notre affaire, p. 204. La "marche à l'étoile» est annonciatrice d'un jour nouveau dans un monde obscurci par les désordres de tous ordres.

16. Une expression qui eût été un bon titre de livre ; Rougemont y avait pensé comme le suggère une note non datée : 1. "Définition de la personne », 2. «Morale du But, ou la vocation», 3. «Avenir du fédéralisme, sa philosophie et ses applications » (Fonds DdR, BPUN, 54.2.3).

17. Note ms. s.d., Fonds DdR, BPUN, 54.2.1.

18. Cf. Tapuscrit, ms. corrigé en 1946 (Fonds DdR, BPUN, 54.1.4). Ce titre a été effleuré par Rougemont, mais il soulève bien des ambiguïtés.

19. Penser avec les mains, p. 203.

20. Ibid., p. 249.

21. Ibid., p. 11.

22. « Destin du siècle ou destin de l'homme » et Penser avec les mains, p. 245-250.

23. Fonds DdR, BPUN, ms., 54.2.2, f. 6.

24. "Le mariage comme décision", un pari, qui impose à chacun des conjoints une "fidélité observée en vertu de l'absurde, parce qu'on s'y est engagé, et que c'est un fait absolu, sur quoi se fonde la personne même des époux " (L'amour et l'Occident, p. 307).

25. Fonds DdR, BPUN, ms., 54.2.2, f. 38.

26. Fonds DdR, BPUN, ms., 54.2.2, f. 39.

27. «La grandeur, l'importance de chacune de nos vies, la dignité que nous attribuons à nos actions, [...] tout cela vient uniquement de la fin à laquelle nous les dédions » (Penser avec les mains, p. 116).

28. Fonds DdR, BPUN, ms., 54.2.2, f. 41.

29. En réalité, la devise jésuite était Ad maiorem Dei gloriam («pour une plus grande gloire de Dieu »).

30. « [...] en matière morale, que de sujets sur lesquels, hélas ! la Bible reste muette ! Quel maigre enseignement elle nous offre sur les grandes questions difficiles avec lesquelles nous nous 
débattons : le mariage, la civilisation, la vie politique » (K. Barth, Parole de Dieu et parole humaine, p. 37).

31. Fonds DdR, BPUN, ms., 54.2.2, f. 56.

32. Rougemont a souvent utilisé l'expression de « désordre établi » pour marquer sa volonté de rompre avec les égarements et les compromissions de son époque.

33. Rougemont cite de mémoire. La formulation exacte et complète est : « Agis de telle sorte que tu traites l'humanité aussi bien dans ta personne que dans la personne de tout autre toujours en même temps comme une fin, et jamais simplement comme un moyen » (cf. E. Kant, Fondements de la métaphysique des mours, p. 150).

34. Cf. K. Barth, «La vocation de l'homme », p. 123 ss. L'on discerne ici une proximité de pensée entre Rougemont et Dietrich Bonhoeffer qui, dans Éthique (1949), consacre des pages denses à la vocation.

35. Le mot se connote d'une signification double. Un sens laïque, en tant qu'il désigne une inclination pour une forme déterminée d'étude ou d'action chez un homme qui possède des aptitudes, des talents correspondants, et un sens religieux, en tant qu'il est une invitation de Dieu à l'homme à accomplir une mission particulière.

36. Fonds DdR, BPUN, ms., 54.2.2, f. 73.

37. «Personne et vocation ne sont point séparables. Et toutes deux ne sont possibles que dans cet acte unique d'obéissance à l'ordre de Dieu, qui s'appelle l'amour du prochain » (Politique de la personne, p. 52). Cf. également «Grammaire de la personne » et « Définition de la personne ».

38. La table des matières provisoire de La morale du but indique clairement que « la définition de la Personne » constituerait la partie conclusive de son essai.

39. Fonds DdR, BPUN, ms., 54.2.2, f. 86.

40. C'est l'une des huit vertus décrite dans ses «Éléments d'une morale de la pensée »: «le style d'une pensée active ", qui «opposera au conformisme la loi personnelle de l'homme, [...] à l'évasion dans l'abstrait la volonté de s'ordonner à un but, et d'y soumettre ses moyens » (Penser avec les mains, p. 228).

41. Fonds DdR, BPUN, ms., 54.2.2, f. 92.

42. Quatorzième petit chapitre de La morale du but, publié in F. Saint-Ouen (éd.), L'Europe de Denis de Rougemont, p. 15-20.

43. Mission ou démission de la Suisse, p. 92.

44. «La vraie défense contre l'esprit totalitaire », p. 425.

45. «Pour une morale de la vocation », p. 29.

\section{RÉSUMÉS}

Manuscrit inédit et inachevé, La morale du but est un essai de philosophie personnaliste inlassablement remis sur le chantier. Face aux désordres de son époque, Denis de Rougemont propose une réflexion qui incite l'homme à donner un sens à sa vie, à inventer une morale personnelle au risque d'une vocation reçue et obéie. 